\title{
Taxonomic Composition and Seasonal Distribution of Copepod Assemblages from Waters Adjacent to Nuclear Power Plant I and II in Northern Taiwan
}

Jiang-Shiou Hwang

Institute of Marine Biology, National Taiwan Ocean University, Keelung, Taiwan 202., Jshwang@mail.ntou.edu.tw

Yueh-Yuan Tu

Department of Industrial Safety and Environmental Protection, Taiwan Power Company, Taipei, Taiwan 100.

Li-Chun Tseng

Institute of Marine Biology, National Taiwan Ocean University, Keelung, Taiwan 202.

Lee-Shing Fang

National Museum of Marine Biology and Aquarium, Pingtung, Taiwan 944.

Sami Souissi

Ecosystem Complexity Research Group, Station Marine de Wimereux, CNRS UMR 8013 ELICO, Universite des Sciences et Technologies de Lille, 28 avenue Foch, 62930 Wimereux, France.

See next page for additional authors

Follow this and additional works at: https://jmstt.ntou.edu.tw/journal

Part of the Terrestrial and Aquatic Ecology Commons

\section{Recommended Citation}

Hwang, Jiang-Shiou; Tu, Yueh-Yuan; Tseng, Li-Chun; Fang, Lee-Shing; Souissi, Sami; Fang, Tien-Hsi; Lo, Wen-Tseng; Twan, Wen-Hung; Hsiao, Shih-Hui; Wu, Cheng-Han; Peng, Shao-Hung; Wei, Tsui-Ping; and Chen, Qing-Chao (2004) "Taxonomic Composition and Seasonal Distribution of Copepod Assemblages from Waters Adjacent to Nuclear Power Plant I and II in Northern Taiwan," Journal of Marine Science and Technology. Vol. 12: Iss. 5, Article 4. DOI: $10.51400 / 2709-6998.2259$

Available at: https://jmstt.ntou.edu.tw/journal/vol12/iss5/4

This Research Article is brought to you for free and open access by Journal of Marine Science and Technology. It has been accepted for inclusion in Journal of Marine Science and Technology by an authorized editor of Journal of Marine Science and Technology. 


\section{Taxonomic Composition and Seasonal Distribution of Copepod Assemblages from Waters Adjacent to Nuclear Power Plant I and II in Northern Taiwan}

\section{Acknowledgements}

We are grateful to Taiwan Power Company and National Museum of Marine Biology and Aquarium, Taiwan, ROC for their financial supports to J. S. Hwang. The authors acknowledge the long term helps from the captain and crews of Ocean Research Vessel II, NSC, Taiwan and sampling and technical helps from the laboratory members, W. S. Liao, C. M. Lin, Y. C. Chang, W. C. Lu, and C. L. Hsu of J.S. Hwang's. We also acknowledge the National Center for Ocean Research providing useful satellite data. We are grateful for the administrative helpers from Taiwan Power Company, C.-S. Tsai, C.-S. Guo, Y.-M. Lin, and G.- K. Chen. We also thank the National Center of Scientific Research (CNRS) of France who supported the scientific stay of J.S. Hwang in France.

\section{Authors}

Jiang-Shiou Hwang, Yueh-Yuan Tu, Li-Chun Tseng, Lee-Shing Fang, Sami Souissi, Tien-Hsi Fang, WenTseng Lo, Wen-Hung Twan, Shih-Hui Hsiao, Cheng-Han Wu, Shao-Hung Peng, Tsui-Ping Wei, and QingChao Chen 


\title{
TAXONOMIC COMPOSITION AND SEASONAL DISTRIBUTION OF COPEPOD ASSEMBLAGES FROM WATERS ADJACENT TO NUCLEAR POWER PLANT I AND II IN NORTHERN TAIWAN
}

\author{
Jiang-Shiou Hwang*, Yueh-Yuan Tu**, Li-Chun Tseng*, Lee-Shing Fang***, \\ Sami Souissi****, Tien-Hsi Fang*****, Wen-Tseng Lo******, Wen-Hung Twan*, \\ Shih-Hui Hsiao*, Cheng-Han Wu*, Shao-Hung Peng*, Tsui-Ping Wei*, \\ and Qing-Chao Chen*******
}

Key words: copepod species composition, copepod distribution, nuclear power plants, thermal discharge.

\section{ABSTRACT}

The nuclear power plants are very important and cheap electric power source for Taiwan. However, the Nuclear Power Plant I and II (NPP I and II) are located in the northern Taiwan where the most populations inhabit. Therefore, the impact of operation of nuclear power plants on the surrounding environment, particularly in the surrounding waters, has drawn great attention to the public of Taiwan. Here we reported the first analyses on a three-year period of monitoring copepod assemblages in the adjacent waters to the NPP I and II. The copepod assemblages in the vicinity waters of NPP I and II were studied from November 2000 to December 2003, and included a total of 13 seasonal sampling cruises. A total of 47 genera, 116 copepod species, including several major genera such as genera of Corycaeus (14 species), Oncaea, Labidocera, Centropages (6 species respectively), and Acartia, Candacia, Oithona (5 species respectively) were identified in the course of the study. Temora turbinata, Calanus sinicus, Canthocalanus pauper, Undinula vulgaris and Paracalanus

Paper Submitted 10/05/04, Accepted 11/26/04. Author for Correspondence: Jiang-Shiou Hwang. E-mail: Jshwang@mail.ntou.edu.tw.

*Institute of Marine Biology, National Taiwan Ocean University, Keelung, Taiwan 202

**Department of Industrial Safety and Environmental Protection, Taiwan Power Company, Taipei, Taiwan 100.

***National Museum of Marine Biology and Aquarium, Pingtung, Taiwan 944.

****Ecosystem Complexity Research Group, Station Marine de Wimereux, CNRS UMR 8013 ELICO, Universite des Sciences et Technologies de Lille, 28 avenue Foch, 62930 Wimereux, France.

***** Department of Oceanography, National Taiwan Ocean University, Keelung, Taiwan 202.

****** Institute of Marine Resources, National Sun Yat-Sen University, Kaohsiung, Taiwan 804.

******* South China Sea Institute of Oceanography, Academia Sinica, Guangzhou, China. aculeatus were the five dominant species, comprising $81 \%$ of the total copepod abundance from sampling stations of NPP I and II during the monitoring period between November 2000 and December 2003. The neritic copepod species Temora turbinata was the most dominant one during all seasons. On the other hand, the continental shelf and oceanic species of Calanus sinicus was very rare during summer and became very dominant (e.g. 19\% of total abundance) in winter indicating the intrusion of cold-water mass from East China Sea.

\section{INTRODUCTION}

Effects of the discharge of the cooling water from nuclear power plants have drawn great attention in the operation of Nuclear Power Plant I and II in northern Taiwan since 1977. The ecological monitoring program has been conducted in the pre-operation, operation and till now. The tremendous amount of long term survey data and findings have been reported [7, 13-15, 31, 4244]. However, due to methodological differences and variations of sampling stations and strategies, relatively less useful data can be made for comparison. In order to establish a standard method and useful data for comparison, a team of investigators has been organized from National Taiwan Ocean University, Academia Sinica and National Sun Yat-Sen University, Taiwan. These team members are responsible for the monitoring program of Nuclear Power Plant I and II since 1998.

The Nuclear Power Plant I and II are located in the northern Taiwan where the most population inhabit. Lin [31] and Hung et al. [15] have found that the thermal discharge from the outlet of NPP II was responsible for the fish body anomalies in the area. However, relatively few copepod data are available since the operation of NPP I and II since 1977 [53]. Wong et al. [53] has identified 37 species of calanoid copepods distributed in the waters of NPP I and NPP II. Among them, 
Acrocalanus gracilis comprising $30 \%$ to $90 \%$ of numeric abundance was the most dominant species in the calanoid copepod assemblages during the sampling period in August 1996 [53]. We present here the first inter-annual study of copepod assemblages obtained from 13 seasonal sampling cruises in waters adjacent to Nuclear Power Plant I and II in Northern Taiwan between November 2000 and December 2003.

Among zooplankton groups, planktonic copepods play a key role in the dynamics of marine ecosystems. They provide a major role in the marine food web from primary producer to higher levels of trophic links. They are also sensitive bio-indicators of environmental change. Therefore, zooplankton, particularly copepoda has been one of the key components in the power plants' cooling water monitoring program $[6,8,38-39,45-46$, $50-51,56]$. Several monitoring programs indicate that thermal stress has a side effect on the zooplankton population. However, little is known about the possible side effect of zooplankton population, particularly copepod assemblages in the waters adjacent to NPP I and NPP II of northern Taiwan. The present study reports the possible side effect of thermal discharge from NPP I and NPP II, northern Taiwan on the copepod assemblages while we compare the copepod assemblages in the surrounding waters of Taiwan.

\section{MATERIALS AND METHODS}

Zooplankton samples were collected by surface net tow at 26 stations in the waters adjacent to Nuclear Power Plant I and II in northern Taiwan (Figure 1). Two $5-\mathrm{km}$ stations also simultaneously collected samples from depth of $30 \mathrm{~m}$. Totally, 28 samples were seasonally collected on board of the Ocean Research Vessel II, NSC, Taiwan. The study covered a 3-year period from November 2000 to December 2003, and included a total of 13 sampling cruises. All zooplankton samples were collected by a Norpac zooplankton net (180 cm long, 45 $\mathrm{cm}$ mouth diameter, $333 \mu \mathrm{m}$ mesh size). Samples were preserved in $5 \%$ buffered formalin. The procedures for sampling, species identification and counting are similar to those described in Hsaio et al. [9], Hwang et al. [16-17], Lo et al. [34, 36], Wong et al. [53], and Wu et al. [55]. All 26 sampling stations with 28 samples were collected on each cruise. The occurrence, relative abundance, average abundance and standard deviation of each species recorded during the monitoring program from November 2000 to December 2003 are computed. In order to study the most dominant species, mean abundance was used to select the species with a contribution greater than $2 \%$. This analysis represented in pie chart was considered for all stations, northern (NPP I) and southern (NPP II) sampling areas and for each season.

\section{RESULTS}

\section{Hydrological structure and water circulation}

Monthly-averaged sea-surface temperature derived from AVHRR for February (Winter), April (Spring), August (Summer) and November (Autumn) of 2002 are presented in Figure 2. The image for February (Figure 2A) shows the intrusion of cold water into the Taiwan Strait and the studied sites of Nuclear Power Plant I and II under the influence of the NE monsoon. Warming of the sea surface temperature to the northern Taiwan was observed in April (Figure 2B). In August, sea surface temperature in the surrounding waters of Taiwan was above $27^{\circ} \mathrm{C}$ (Figure 2C). Onset of the NE monsoon and cold water intrusion from the north was observed in November (Figure 2D). Sea surface temperature to the east and southeast of Taiwan was above $24^{\circ} \mathrm{C}$ throughout the year due to the influence of the Kuroshio Current.

\section{Copepod assemblages in the waters of Nuclear Power Plant I and II}

A total of 47 genera, 116 copepod species, including several major genera such as genera of Corycaeus (14 species), Oncaea, Labidocera, Centropages (6 species respectively), and Acartia, Candacia, Oithona (5 species respectively) were found in the course of the

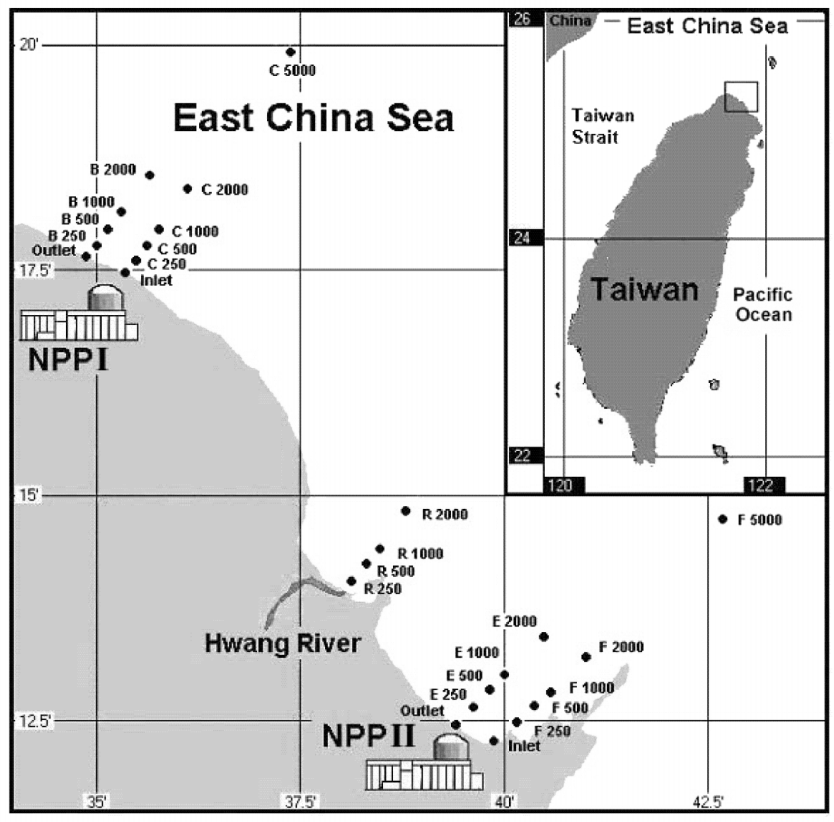

Fig. 1. Map and location of sampling stations in Nuclear Power Plant I and II (NPP I and II), in northern Taiwan. 
study (Table 1). Temora turbinata, Calanus sinicus, Canthocalanus pauper, Undinula vulgaris and Paracalanus aculeatus were the five dominant species, comprising $81 \%$ of the total copepod numbers from all stations of NPP I and II during the monitoring period between November 2000 and December 2003 (Figure 3 ). The dominant copepod species varied widely among seasons (Table 2). However, Temora turbinata was the

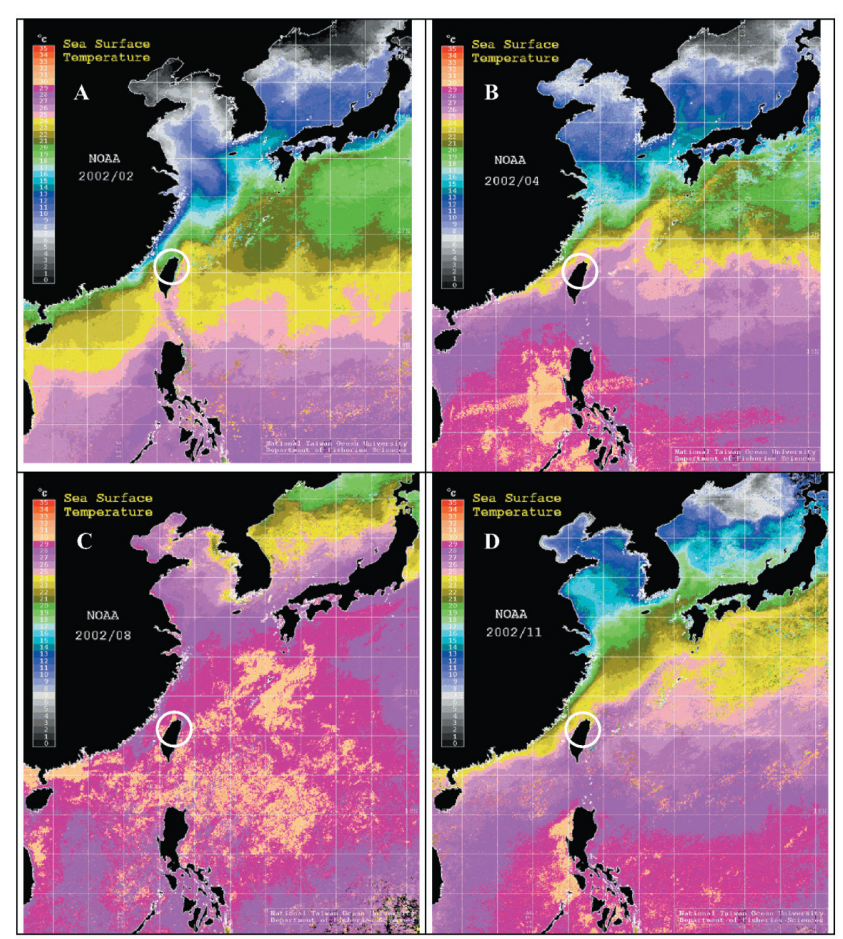

Fig. 2. Monthly-averaged sea-surface temperature derived from AVHRR for February (A), April (B), August (C) and November (D) 2002. The white circle in each figure marks the northern part of Taiwan.

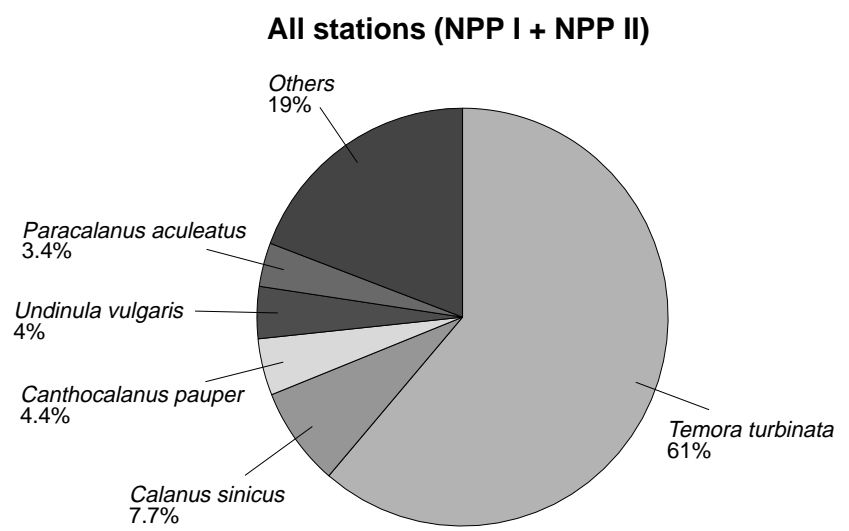

Fig. 3. Pie chart representing the most dominant species in all stations during the studying period between November 2000 and December 2003. most dominant species in all stations (Figure 3) both NPP I (Figure 4) and NPP II (Figure 5). Temora turbinata was the most dominant species in all seasons during spring e.g. 59\% (Figure 6), summer e.g. 77\% (Figure 7), autumn e.g. 32\% (Figure 8) and winter e.g. $21 \%$ (Figure 9). Calanus sinicus reached the highest density during winter e.g. 19\% (Figure 9) and started declining in spring e.g. 14\% (Figure 6). Calanus sinicus was in low density during autumn and summer (Table 2, Figure 7 and Figure 8).

\section{DISCUSSION}

The island of Taiwan is influenced by several major ocean currents and water masses [27, 29-30, 32, $47,54]$. These ocean currents and water masses are important sources to introduce a large numbers of cope-

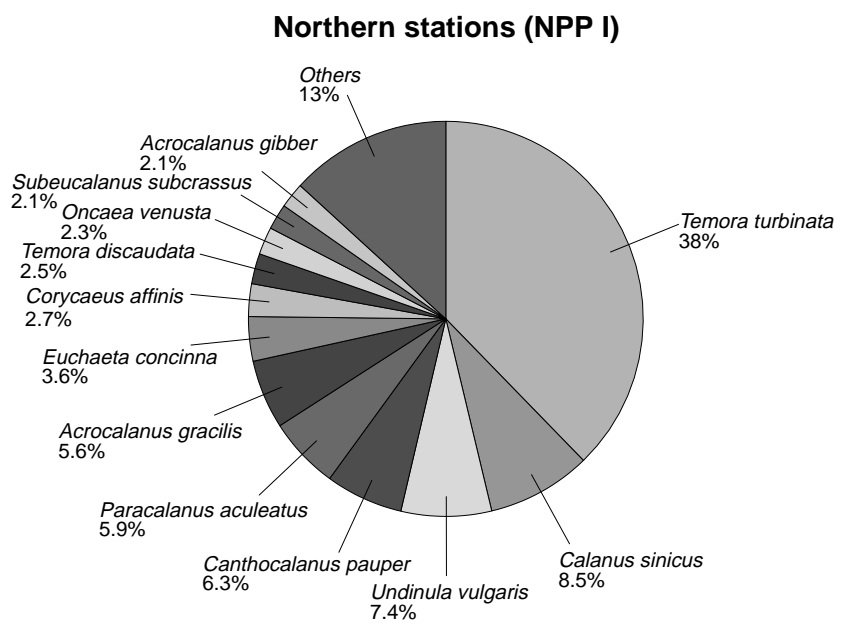

Fig. 4. Pie chart representing the most dominant species in all stations of NPP I during the studying period between November 2000 and December 2003.

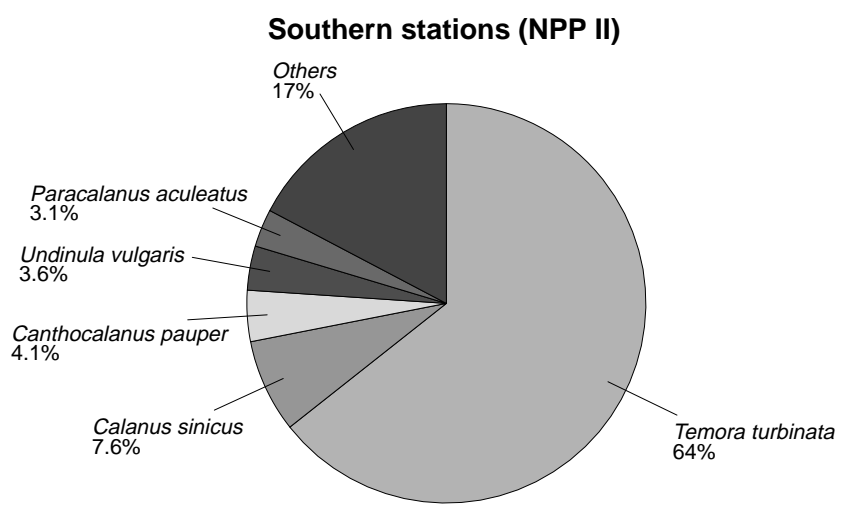

Fig. 5. Pie chart representing the most dominant species in all stations of NPP II during the studying period between November 2000 and December 2003. 
Table 1. The list of copepod species recorded during the monitoring program from 2000 to 2003 in 26 stations off Nuclear Power Plant in the northern Taiwan. The occurrence $(\mathrm{OR}, \%)$, relative abundance $(\mathrm{RA}, \%)$, average abundance (Mean, individuals/ $10^{3} \mathrm{~m}^{3}$ ) and standard deviation (individuals $\mathrm{m}^{-3}$ )

\begin{tabular}{|c|c|c|c|c|}
\hline Species name & OR & $\mathbf{R A}$ & Mean & SD \\
\hline \multicolumn{5}{|l|}{ Acartiidae } \\
\hline Acartia (Odontacartia) erythraea Giesbrecht 1889 & 6.04 & 0.0300 & 91.90 & 473.49 \\
\hline Acartia (Odontacartia) pacifica Giesbrecht 1888 & 5.77 & 0.0250 & 76.58 & 444.88 \\
\hline Acartia (Odontacartia) spinicauda Giesbrecht 1889 & 9.34 & 0.1919 & 587.80 & $5,270.56$ \\
\hline Acartia (Plantacartia) danae, Giesbrecht 1889 & 0.27 & 0.0001 & 0.18 & 3.35 \\
\hline Acartia (Plantacartia) negligens Dana 1849 & 37.36 & 0.4191 & $1,283.56$ & $5,413.98$ \\
\hline \multicolumn{5}{|l|}{ Aetideidae } \\
\hline \multirow{2}{*}{\multicolumn{5}{|c|}{ Arietellidae }} \\
\hline & & & & \\
\hline Metacalanus aurivillii, Cleve 1901 & 0.27 & 0.0002 & 0.51 & 9.64 \\
\hline \multicolumn{5}{|l|}{ Augaptilidae } \\
\hline Haloptilus longicornis, (Claus) 1863 & 0.27 & 0.0000 & 0.03 & 0.58 \\
\hline \multicolumn{5}{|l|}{ Calanidae } \\
\hline Calanoides carinatus (Kroeyer) 1849 & 3.02 & 0.0226 & 69.07 & 510.61 \\
\hline Calanus sinicus Brodsky 1965 & 42.58 & 7.6743 & $23,505.67$ & $100,720.70$ \\
\hline Canthocalanus pauper (Giesbrecht) 1888 & 73.63 & 4.3928 & $13,454.75$ & $43,507.57$ \\
\hline Cosmocalanus darwini (Lubbock) 1860 & 20.88 & 0.3506 & $1,073.73$ & $5,115.78$ \\
\hline Mesocalanus tenuicornis, (Dana) 1863 & 0.27 & 0.0000 & 0.12 & 2.31 \\
\hline Nannocalanus minor (Claus) 1863 & 7.97 & 0.0759 & 232.60 & $1,760.27$ \\
\hline Undinula vulgaris (Dana) 1849 & 56.87 & 4.0240 & $12,325.32$ & $36,083.74$ \\
\hline \multicolumn{5}{|l|}{ Calocalanidae } \\
\hline Calocalanus pavo (Dana) 1849 & 5.22 & 0.1020 & 312.55 & $2,029.61$ \\
\hline Calocalanus pavoninus Farran 1936 & 0.82 & 0.0035 & 10.85 & 187.36 \\
\hline Calocalanus plumulosus (Claus) 1863 & 1.10 & 0.0048 & 14.76 & 187.49 \\
\hline \multicolumn{5}{|l|}{ Candaciidae } \\
\hline Candacia bradyi A. Scott 1902 & 6.04 & 0.0682 & 208.91 & $2,201.87$ \\
\hline Candacia catula (Giesbrecht) 1889 & 3.57 & 0.0192 & 58.93 & 440.98 \\
\hline Candacia discaudata, A. Scott 1909 & 0.27 & 0.0020 & 6.04 & 115.26 \\
\hline Candacia ethiopica (Dana) 1849 & 2.20 & 0.0064 & 19.68 & 179.33 \\
\hline Candacia pachydactyla (Dana) 1849 & 4.12 & 0.0106 & 32.43 & 203.90 \\
\hline Paracandacia truncata (Dana) 1849 & 4.67 & 0.0579 & 177.21 & $1,486.94$ \\
\hline \multicolumn{5}{|l|}{ Centropagedae } \\
\hline Centropages calaninus (Dana) 1849 & 3.85 & 0.0205 & 62.90 & 440.79 \\
\hline Centropages furcatus (Dana) 1849 & 22.80 & 0.2311 & 707.77 & 2,529.62 \\
\hline \multicolumn{5}{|l|}{ Centropages gracilis (Dana) 1849} \\
\hline Centropages orsini Giesbrecht 1889 & 9.07 & 0.2199 & 673.45 & $5,366.55$ \\
\hline Centropages sinensis, Chen and Zhang 1965 & 1.10 & 0.0009 & 2.63 & 27.34 \\
\hline Centropages tenuiremis Thompson and Scott 1903 & 2.20 & 0.0149 & 45.59 & 468.18 \\
\hline \multicolumn{5}{|l|}{ Clausocalanidae } \\
\hline Clausocalanus arcuicornis (Dana) 1849 & 39.01 & 1.2756 & $3,907.09$ & $45,053.56$ \\
\hline Clausocalanus furcatus (Brady) 1883 & 22.53 & 0.2852 & 873.62 & $3,227.95$ \\
\hline Clausocalanus mastigophorus (Claus) 1863 & 16.76 & 0.1640 & 502.21 & $1,785.20$ \\
\hline \multicolumn{5}{|l|}{ Clytemnestridae } \\
\hline Clytemnestra scutellata Dana 1847 & 0.82 & 0.0004 & 1.37 & 15.19 \\
\hline \multicolumn{5}{|l|}{ Corycaeidae } \\
\hline Corycaeus (Agetus) flaccus, Giesbrecht 1891 & 1.37 & 0.0073 & 22.34 & 300.44 \\
\hline Corycaeus (Corycaeus) crassiusculus, Dana 1849 & 1.10 & 0.0007 & 2.15 & 21.29 \\
\hline Corycaeus (Corycaeus) speciosus, Dana 1849 & 6.59 & 0.0284 & 87.02 & 499.99 \\
\hline Corycaeus (Ditrichocorycaeus) affinis, McMurrich 1916 & 37.09 & 0.7642 & $2,340.55$ & $9,988.86$ \\
\hline
\end{tabular}




\begin{tabular}{|c|c|c|c|c|}
\hline Corycaeus (Ditrichocorycaeus) andrewsi, Farran 1911 & 5.77 & 0.0635 & 194.50 & $1,368.2 \varepsilon$ \\
\hline Corycaeus (Ditrichocorycaeus) asiaticus, F. Dahl 1894 & 4.12 & 0.0096 & 29.31 & 216.3 \\
\hline Corycaeus (Ditrichocorycaeus) dahli, Tanaka 1957 & 15.38 & 0.1090 & 333.97 & $2,196.62$ \\
\hline Corycaeus (Ditrichocorycaeus) erythraeus, Cleve 1901 & 8.52 & 0.0302 & 92.60 & 465.9( \\
\hline Corycaeus (Farranula) concinna, (Dana) 1847 & 0.55 & 0.0011 & 3.45 & 57.0 \\
\hline Corycaeus (Farranula) gibbula, Giesbrecht, 1891 & 9.62 & 0.0717 & 219.65 & $1,051.3$ \\
\hline Corycaeus (Onychocorycaeus) agilis, Dana 1849 & 3.57 & 0.0091 & 27.74 & 224.17 \\
\hline Corycaeus (Onychocorycaeus) catus, F. Dahl 1894 & 8.52 & 0.0659 & 201.88 & $2,184.66$ \\
\hline Corycaeus (Onychocorycaeus) pacificus, M. Dahl 1912 & 5.77 & 0.0420 & 128.75 & 688.5 \\
\hline Corycaeus (Onychocorycaeus) pumilus, M. Dahl 1912 & 13.19 & 0.0493 & 150.90 & 573.6 \\
\hline Corycaeus (Urocorycaeus) lautus, Dana 1849 & 1.92 & 0.0091 & 27.94 & 250.5 \\
\hline Corycaeus (Urocorycaeus) longistylis, Dana 1849 & 4.12 & 0.0078 & 23.89 & 210.0 \\
\hline \multicolumn{5}{|l|}{ Eucalanidae } \\
\hline Pareucalanus attenuatus (Dana) 1849 & 6.04 & 0.0460 & 140.91 & 759.0 \\
\hline Rhincalanus nasutus Giesbrecht 1888 & 0.82 & 0.0051 & 15.63 & 205.9 \\
\hline Rhincalanus rostrifrons (Dana) 1852 & 2.20 & 0.0091 & 27.73 & 243.8 \\
\hline Subeucalanus crassus (Giesbrecht) 1888 & 19.78 & 0.2544 & 779.34 & $3,213.3$ \\
\hline Subeucalanus pileatus (Giesbrecht) 1888 & 7.42 & 0.2268 & 694.60 & $3,496.5$ \\
\hline Subeucalanus subcrassus (Giesbrecht) 1888 & 67.58 & 1.5374 & $4,708.79$ & $10,819.5$ \\
\hline Subeucalanus subtenuis (Giesbrecht) 1888 & 12.64 & 0.0962 & 294.52 & $1,246.4$ \\
\hline \multicolumn{5}{|l|}{ Euchaetidae } \\
\hline Euchaeta concinna (Dana) 1849 & 29.95 & 0.9799 & $3,001.40$ & $11,583$. \\
\hline Euchaeta indica Wolfenden 1905 & 12.09 & 0.2246 & 687.82 & 2,988 \\
\hline Euchaeta plana Mori 1937 & 10.16 & 0.2651 & 812.02 & 3,769 . \\
\hline Euchaeta rimana Bradford 1973 & 18.68 & 0.4451 & $1,363.18$ & $7,788.22$ \\
\hline Paraeuchaeta russelli (Farran) 1936 & 6.87 & 0.3563 & $1,091.43$ & $7,186.5$ \\
\hline \multicolumn{5}{|l|}{ Euterpinidae } \\
\hline Euterpina acutifrons (Dana) 1847 & 3.30 & 0.0114 & 34.84 & 303.2 \\
\hline \multicolumn{5}{|l|}{ Heterorhabdidae } \\
\hline Heterorhabdus papilliger (Claus) 1863 & 0.55 & 0.0052 & 15.95 & 217.9 \\
\hline \multicolumn{5}{|l|}{ Lucicutiidae } \\
\hline Lucicutia clausi, (Giesbrecht) 1889 & 0.27 & 0.0015 & 4.62 & 88.16 \\
\hline Lucicutia flavicornis (Claus) 1863 & 4.67 & 0.0174 & 53.25 & 394.5( \\
\hline Lucicutia ovalis (Giesbrecht) 1889 & 0.82 & 0.0016 & 4.96 & \\
\hline \multicolumn{5}{|l|}{ Mecynoceridae } \\
\hline Mecynocera clausi Thompson 1888 & 0.55 & 0.0006 & 1.75 & 24.9 \\
\hline \multicolumn{5}{|l|}{ Metridinidae } \\
\hline Pleuromamma abdominalis (Lubbock) 1856 & 0.27 & 0.0002 & 0.74 & 14.15 \\
\hline Pleuromamma gracilis (Claus) 1863 & 2.47 & 0.0304 & 93.09 & $1,169.97$ \\
\hline \multicolumn{5}{|l|}{ Miraciidae } \\
\hline Macrosetella gracilis (Dana) 1847 & 5.49 & 0.0108 & 33.14 & 234.7 \\
\hline \multicolumn{5}{|l|}{ Oithonidae } \\
\hline Oithona attenuata Farran 1913 & 1.92 & 0.0047 & 14.44 & 155.2( \\
\hline Oithona fallax Farran 1913 & 0.82 & 0.0006 & 1.86 & 21.2( \\
\hline Oithona rigida Giesbrecht 1896 & 6.04 & 0.0350 & 107.33 & 685.6 \\
\hline Oithona setigera (Dana) 1849 & 18.13 & 0.1308 & 400.70 & $1,662.7$ \\
\hline Oithona similis Claus 1866 & 0.55 & 0.0001 & 0.43 & 7.47 \\
\hline \multicolumn{5}{|l|}{ Oncaeidae } \\
\hline Oncaea clevei Fruhtl 1863 & 0.82 & 0.0002 & 0.67 & \\
\hline Oncaea conifera Giesbrecht 1891 & 27.75 & 0.3862 & $1,182.94$ & $7,036.46$ \\
\hline Oncaea media Giesbrecht 1891 & 7.97 & 0.0305 & 93.31 & 581.8 \\
\hline Oncaea mediterranea Claus 1861 & 0.82 & 0.0076 & 23.39 & 264.71 \\
\hline Oncaea similis Sars 1918 & 4.95 & 0.0149 & 45.76 & 338.86 \\
\hline Oncaea venusta Philippi 1843 & 53.02 & 0.9902 & $3,032.94$ & $9,710.16$ \\
\hline
\end{tabular}




\section{Paracalanidae}

Acrocalanus gibber Giesbrecht 1888

Acrocalanus gracilis Giesbrecht 1888

Acrocalanus monachus Giesbrecht 1888

Paracalanus aculeatus Giesbrecht 1888

Paracalanus gracilis, Chen and Zhang 1965

Paracalanus nanus Sars 1907

Paracalanus nudus Sewell, 1929

Paracalanus parvus (Claus) 1863

Parvocalanus crassirostris (Dahl) 1893

\section{Pontellidae}

Calanopia elliptica (Dana) 1849

Calanopia minor A. Scott 1902

Labidocera acuta (Dana) 1849

Labidocera bipinnata Tanaka 1936

Labidocera detruncata (Dana) 1849

Labidocera euchaeta Giesbrecht 1889

Labidocera kroeyeri (Brady) 1883

Labidocera minuta Giesbrecht 1889

Pontella chierchiae, Giesbrecht 1889

Pontella fera Dana 1849

Pontella securifer, Brady 1883

Pontella sinica, Chen and Zhang 1965

Pontellina plumata (Dana) 1849

Pontellopsis regalis (Dana) 1849

Pontellopsis tenuicauda (Giesbrecht) 1889

Pontellopsis yamadae Mori 1937

Pseudodiaptomidae

Pseudodiaptomus annandalei Sewell 1919

\section{Sapphirinidae}

Copilia mirabilis Dana 1849

Sapphirina darwini Haeckel 1864

Sapphirina nigromaculata Claus 1863

Sapphirina scarlata, Giesbrecht 1891

\section{Scolecithricidae}

Scolecithricella longispinosa Chen and Zhang 1965

Scolecithrix danae (Lubbock) 1856

\section{Temoridae}

Temora discaudata (Giesbrecht) 1889

Temora turbinata (Dana) 1849

\begin{tabular}{|c|c|c|c|}
\hline 40.38 & 1.8731 & $5,737.17$ & $22,235.80$ \\
\hline 40.11 & 1.6799 & $5,145.33$ & $19,623.22$ \\
\hline 4.67 & 0.0197 & 60.47 & 544.49 \\
\hline 66.21 & 3.3921 & $10,389.69$ & $31,077.11$ \\
\hline 7.14 & 0.3455 & $1,058.27$ & $6,565.89$ \\
\hline 6.32 & 0.0903 & 276.65 & $2,525.76$ \\
\hline 0.82 & 0.0014 & 4.41 & 69.02 \\
\hline 19.78 & 0.5724 & $1,753.34$ & $13,364.96$ \\
\hline 4.40 & 0.0635 & 194.54 & $1,735.61$ \\
\hline 19.51 & 0.3720 & $1,139.44$ & $5,345.38$ \\
\hline 5.49 & 0.0959 & 293.85 & $3,279.90$ \\
\hline 16.48 & 0.4861 & $1,488.86$ & $9,450.77$ \\
\hline 3.57 & 0.0161 & 49.26 & 412.75 \\
\hline 3.02 & 0.0156 & 47.77 & 416.22 \\
\hline 10.71 & 0.0725 & 221.93 & $1,408.15$ \\
\hline 5.77 & 0.0199 & 61.00 & 354.80 \\
\hline 14.01 & 0.2606 & 798.23 & $4,528.80$ \\
\hline 1.10 & 0.0015 & 4.62 & 58.91 \\
\hline 0.55 & 0.0005 & 1.44 & 26.64 \\
\hline 0.27 & 0.0004 & 1.12 & 21.39 \\
\hline 0.27 & 0.0002 & 0.66 & 12.63 \\
\hline 3.57 & 0.0135 & 41.26 & 264.88 \\
\hline 0.82 & 0.0024 & 7.49 & 116.54 \\
\hline 1.37 & 0.0056 & 17.25 & 181.34 \\
\hline 2.47 & 0.0142 & 43.63 & 481.95 \\
\hline 0.27 & 0.0004 & 1.28 & 24.37 \\
\hline 1.92 & 0.0121 & 37.05 & 306.00 \\
\hline 0.27 & 0.0024 & 7.45 & 142.20 \\
\hline 0.27 & 0.0025 & 7.69 & 146.66 \\
\hline 0.82 & 0.0047 & 14.44 & 159.00 \\
\hline 14.84 & 0.1296 & 396.80 & $1,655.99$ \\
\hline 10.44 & 0.2999 & 918.65 & $11,524.41$ \\
\hline 54.95 & 1.7718 & $5,426.96$ & $14,373.00$ \\
\hline 87.91 & 61.2723 & $187,672.11$ & $819,416.82$ \\
\hline
\end{tabular}

pod species and maintain high copepod diversity in the surrounding waters of Taiwan. To the east of Taiwan, a year-round northward flowing of the Kuroshio Current generated the high copepod diversity of the region. To the north of Taiwan, the NE monsoon is the primary driving force for the possible introduction of copepod species along Chinese coast from the Bohai Sea and the East China Sea into the Taiwan Strait during the winter $[2,26]$. To the south of Taiwan, the SW monsoon provides another possible pathway for the introduction of copepod species from South China Sea during sum- mer [2]. The copepod fauna of Taiwan was enriched by the introduction of temperate and subtropical species from the north and tropical species from the south. Based on previously published reviews, taxonomists have estimated that the waters of Taiwan may contain about $10 \%$ of the species of the global marine fauna [25]. Shih and Young [41] have reviewed the published records of 431 species of copepods occurring in the marginal seas of China, including the surrounding waters of Taiwan. This phenomenon particularly pronounced in the upwelling water of northern Taiwan. In 
Table 2. Average abundance (mean \pm SD) and relative abundance of the top five dominant copepod species recorded during each sampling cruise

\begin{tabular}{|c|c|c|c|c|}
\hline \multirow[t]{2}{*}{ Sampling Cruise } & \multirow[t]{2}{*}{ Species } & \multicolumn{2}{|c|}{ Average abundance } & \multirow{2}{*}{$\begin{array}{c}\text { Relative abundance } \\
(\%)\end{array}$} \\
\hline & & $\left(\right.$ ind.$\left.m^{-3}\right)$ & $( \pm \mathrm{SD})$ & \\
\hline \multirow[t]{5}{*}{ November 2000} & Temora turbinata & 45.16 & 60.80 & 38.14 \\
\hline & Euchaeta concinna & 8.19 & 14.24 & 6.91 \\
\hline & Acrocalanus gibber & 7.96 & 9.32 & 6.72 \\
\hline & Undinula vulgaris & 7.41 & 9.70 & 6.26 \\
\hline & Canthocalanus pauper & 7.32 & 11.88 & 6.18 \\
\hline \multirow[t]{5}{*}{ March 2001} & Calanus sinicus & 49.25 & 70.03 & 29.69 \\
\hline & Temora turbinata & 24.73 & 36.00 & 14.91 \\
\hline & Canthocalanus pauper & 21.61 & 25.16 & 13.03 \\
\hline & Euchaeta concinna & 11.94 & 32.29 & 7.20 \\
\hline & Paracalanus aculeatus & 10.41 & 14.50 & 6.28 \\
\hline \multirow[t]{5}{*}{ May 2001} & Temora turbinata & 758.73 & $1,322.63$ & 80.59 \\
\hline & Undinula vulgaris & 39.58 & 87.97 & 4.20 \\
\hline & Canthocalanus pauper & 19.17 & 34.34 & 2.04 \\
\hline & Labidocera acuta & 14.38 & 31.34 & 1.53 \\
\hline & Subeucalanus subcrassus & 11.74 & 20.18 & 1.25 \\
\hline \multirow[t]{5}{*}{ August 2001} & Acrocalanus gibber & 41.76 & 60.04 & 30.50 \\
\hline & Canthocalanus pauper & 21.50 & 40.53 & 15.70 \\
\hline & Acrocalanus gracilis & 17.57 & 34.53 & 12.83 \\
\hline & Undinula vulgaris & 14.21 & 33.31 & 10.37 \\
\hline & Subeucalanus subcrassus & 8.54 & 15.46 & 6.24 \\
\hline \multirow[t]{5}{*}{ October 2001} & Temora turbinata & 57.93 & 214.90 & 27.81 \\
\hline & Canthocalanus pauper & 42.43 & 127.05 & 20.37 \\
\hline & Clausocalanus arcuicornis & 33.17 & 161.54 & 15.92 \\
\hline & Acrocalanus gibber & 14.21 & 32.77 & 6.82 \\
\hline & Paracalanus aculeatus & 11.44 & 24.74 & 5.49 \\
\hline \multirow[t]{5}{*}{ March 2002} & Temora turbinata & 282.05 & 423.41 & 87.87 \\
\hline & Calanus sinicus & 16.52 & 28.64 & 5.15 \\
\hline & Corycaeus (Ditrichocorycaeus) affinis & 7.66 & 23.61 & 2.39 \\
\hline & Canthocalanus pauper & 4.31 & 7.14 & 1.34 \\
\hline & Subeucalanus subcrassus & 1.64 & 2.66 & 0.51 \\
\hline \multirow[t]{5}{*}{ May 2002} & Temora discaudata & 24.68 & 32.70 & 34.02 \\
\hline & Paraeuchaeta russelli & 14.18 & 22.40 & 19.54 \\
\hline & Subeucalanus pileatus & 9.03 & 9.29 & 12.44 \\
\hline & Calanopia minor & 3.50 & 11.51 & 4.82 \\
\hline & Euchaeta indica & 3.38 & 4.87 & 4.65 \\
\hline \multirow[t]{5}{*}{ July 2002} & Temora turbinata & 652.58 & $2,376.26$ & 80.50 \\
\hline & Canthocalanus pauper & 37.56 & 54.42 & 4.63 \\
\hline & Acrocalanus gracilis & 37.16 & 48.12 & 4.58 \\
\hline & Undinula vulgaris & 32.98 & 47.73 & 4.07 \\
\hline & Oncaea venusta & 8.72 & 17.39 & 1.08 \\
\hline \multirow[t]{5}{*}{ October 2002} & Temora turbinata & 22.40 & 54.50 & 31.70 \\
\hline & Undinula vulgaris & 20.17 & 41.97 & 28.54 \\
\hline & Acartia (Odontacartia) spinicauda & 7.50 & 17.88 & 10.62 \\
\hline & Paracalanus aculeatus & 7.03 & 21.27 & 9.95 \\
\hline & Subeucalanus subcrassus & 2.82 & 4.65 & 3.99 \\
\hline \multirow[t]{5}{*}{ January 2003} & Calanus sinicus & 25.94 & 33.01 & 28.30 \\
\hline & Paracalanus aculeatus & 23.31 & 25.60 & 25.43 \\
\hline & Clausocalanus arcuicornis & 6.20 & 7.71 & 6.76 \\
\hline & Temora turbinata & 5.30 & 6.06 & 5.78 \\
\hline & Clausocalanus furcatus & 4.30 & 7.23 & 4.69 \\
\hline \multirow[t]{2}{*}{ April 2003} & Calanus sinicus & 203.95 & 300.30 & 51.01 \\
\hline & Paracalanus aculeatus & 49.71 & 85.15 & 12.43 \\
\hline
\end{tabular}


July 2003

Temora turbinata
Paracalanus parvus
Temora discaudata
Temora turbinata
Undinula vulgaris
ubeucalanus subcrassus
Canthocalanus pauper
Acrocalanus gracilis
Temora turbinata
Paracalanus gracilis
Calanus sinicus
Undinula vulgaris

Clausocalanus arcuicornis

$\begin{array}{rrr}46.05 & 43.03 & 11.52 \\ 16.49 & 45.59 & 4.12 \\ 15.86 & 22.42 & 3.97 \\ 505.58 & 686.63 & 89.74 \\ 24.04 & 45.21 & 4.27 \\ 8.56 & 12.40 & 1.52 \\ 6.81 & 11.68 & 1.21 \\ 3.26 & 8.03 & 0.58 \\ 31.16 & 54.09 & 38.41 \\ 13.76 & 19.96 & 16.96 \\ 6.31 & 9.97 & 7.77 \\ 5.00 & 9.36 & 6.16 \\ 3.54 & 7.35 & 4.36\end{array}$

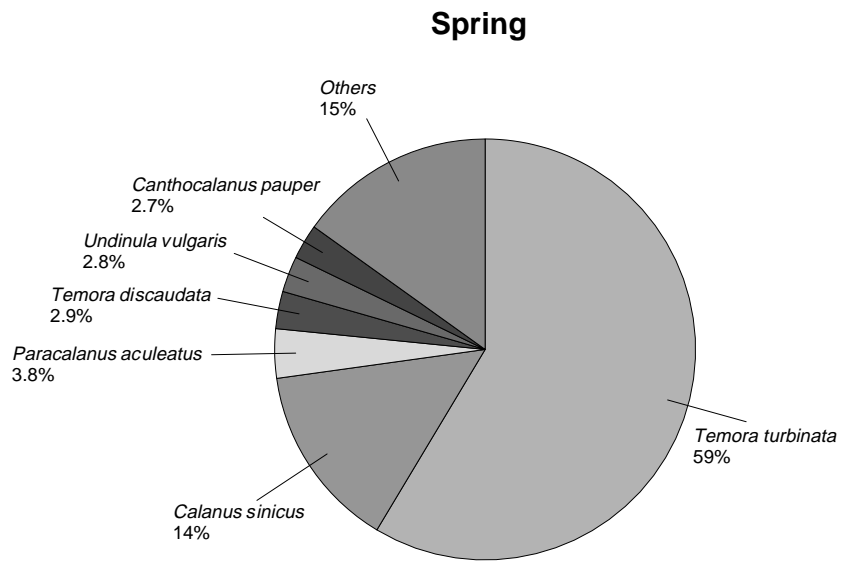

Fig. 6. Pie chart representing the most dominant species in all stations of NPP I and II during the studying period between November 2000 and December 2003 in spring.

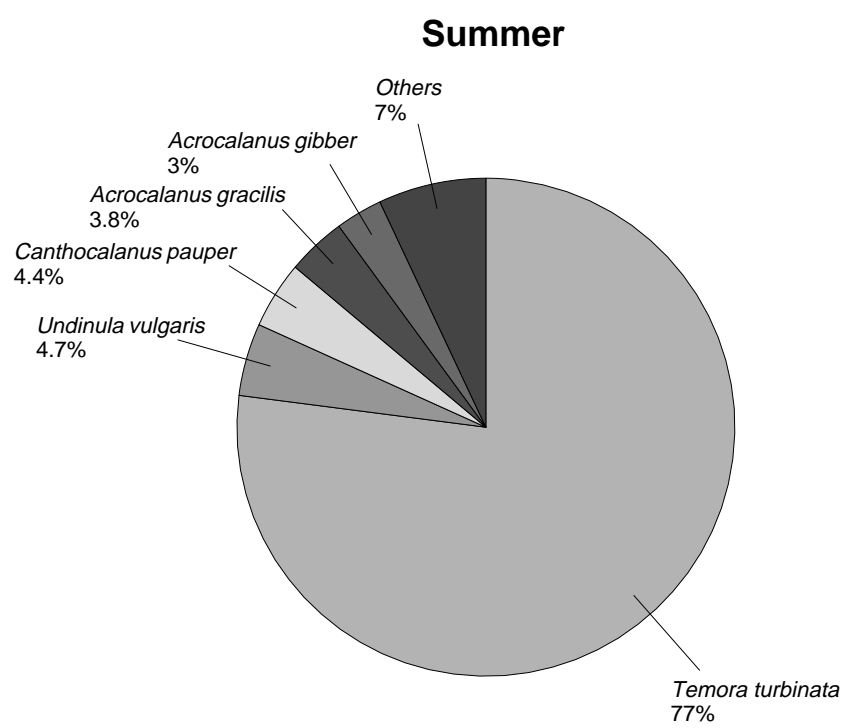

Fig. 7. Pie chart representing the most dominant species in all stations of NPP I and II during the studying period between November 2000 and December 2003 in summer.

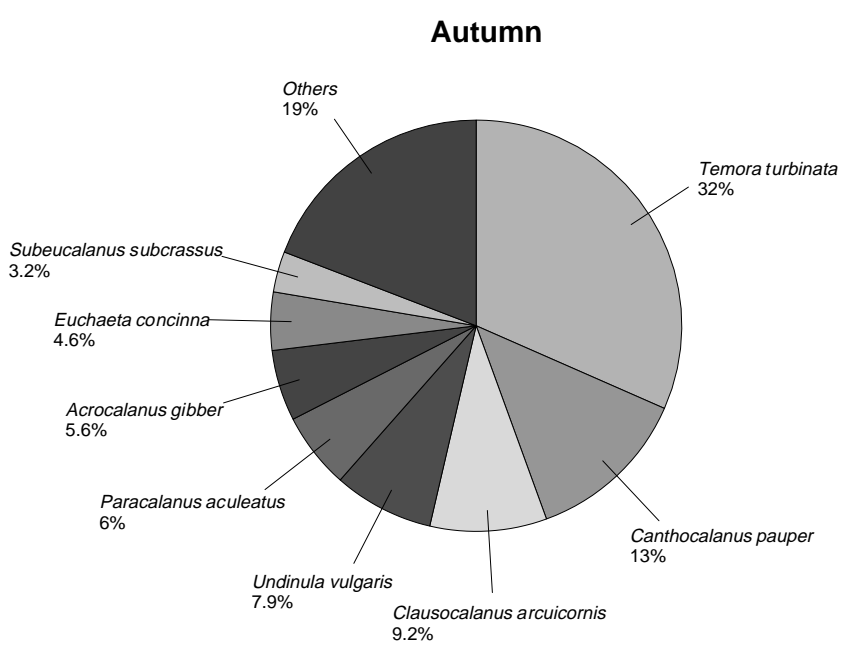

Fig. 8. Pie chart representing the most dominant species in all stations of NPP I and II during the studying period between November 2000 and December 2003 in autumn.

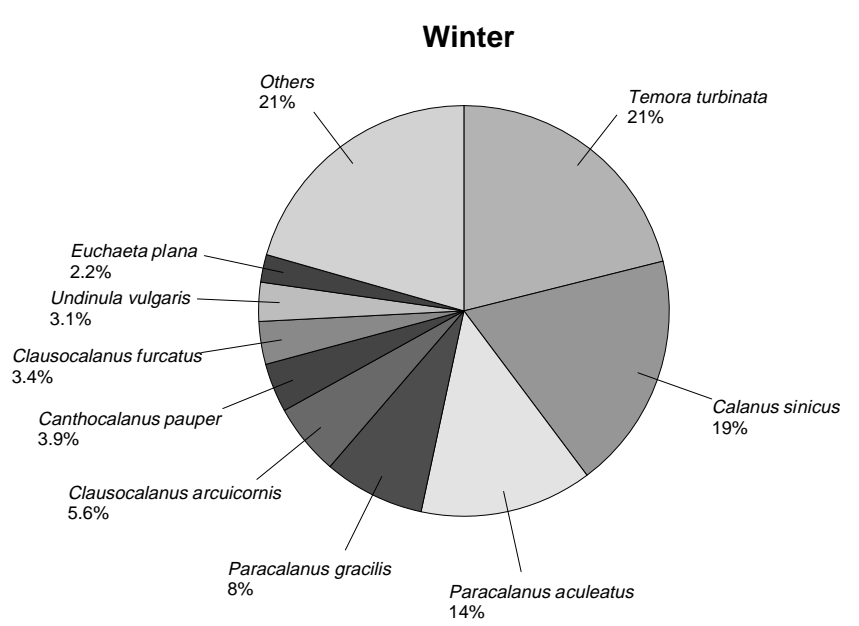

Fig. 9. Pie chart representing the most dominant species in all stations of NPP I and II during the studying period between November 2000 and December 2003 in winter. 
1995, a total of 178 copepod species were identified in the upwelling water of the Mienhua Canyon, northern Taiwan [36]. In the present 3-year study, a total of 47 genera, 116 copepod species was found in the adjacent waters of Nuclear Power Plant I and II (Table 1). Several major genera such as genera of Corycaeus (14 species), Oncaea, Labidocera, Centropages (6 species respec-tively), and Acartia, Candacia, Oithona (5 species re-spectively) were identified. Temora turbinata, Calanus sinicus, Canthocalanus pauper, Undinula vulgaris and Paracalanus aculeatus were the five dominant species, comprising $81 \%$ of the total copepod numbers (Table 1) from all stations of NPP I and II (Figure 3) during the monitoring period between November 2000 and December 2003. Interestingly, Paracalanus aculeatus and Canthocalanus pauper were the common dominant species both in the upwelling water during early spring [36] and waters adjacent to Nuclear Power Plant I and II (Figure 3) in total averaged stations and seasons. However, the common dominant species of Temora discaudata and Oncaea venusta reported by Lo et al. (36) and present study are only common occurred in NPP I but not in NPP II. Several common dominant species also varied through time. The dominant species reported by Lo et al. [36] and present study of Temora discaudata in spring, Clausocalanus arcuicornis and C. furcatus in winter respectively are common in abundance. This indicated that there were some similarity of copepod assemblages in both Lo et al. (36) upwelling water and present study of coastal waters adjacent to NPP I and NPP II. Apparently, the discharge of cooling water from NPP I and NPP II did not significant change the copepod assemblages while both sampling sites were located in the northern Taiwan. It should be noticed that the dominant species of Calanus sinicus in the waters adjacent to NPP I and II usually occurred in high abundance during the winter and spring while the cold water mass from East China Sea (Figure 2A) intruded into the coastal waters of northern Taiwan. The high seawater temperature is not suitable for Calanus sinicus to growth and survivor [26, 37-38, 48, 52]. The seawater temperature and food availability play a key role in the development, energy conservation and life history strategies of Calanus sinicus [37-38]. The seawater temperature of Kuroshio current usually is sub-lethal or lethal to Calanus sinicus [26, 49]. Therefore, Calanus sinicus was not the dominant species in the Lo et al. [36] sampling station where the location is surrounded by waters from Kuroshio Current. Several studies also indicated that the occurrence of Calanus sinicus was seawater temperature dependent [16-17, 37-38, 52]. Calanus sinicus is common in spring and winter in the present study and some waters of Taiwan during spring
$[16,28]$ and winter [17] while these seawater temperatures are relatively low and is suitable for Calanus sinicus to survive. Usually, Calanus sinicus was transported by the intrusion of cold-water mass from its population centre of Bohai Sea, Yellow Sea and East China Sea [26]. However, it is rare during summer [53] and present study (Tables 1 and 2). Wang et al. [52] discovered that Calanus sinicus migrated to bottom cold water for over-summer while shallow seawater temperature was high. According to the review of Shih and Young [41], there are 325 species of copepods in the East China Sea region. Therefore, it indicated that most of the copepod species in the East China Sea region probably belong to rare species. Results presented here support this suggestion. The five more abundant species are accounted for about $81 \%$ of the total copepod abundance and most of the other species occurred in very low density (Table 1 ).

In the past decade, most published papers on copepod biology and ecology from Taiwan have mainly focused on short-term studies (e.g., References 1, 3-5, 9-12, 16-17, 24-25, 28, 33-36, 40, and 55). Very little of the copepod studies has focused on long-term studies of spatial and temporal distribution of copepods from Taiwan. This long-term study provides important information on copepod species composition (Table 1) and temporal distribution of dominant species (Table 2, Figures 3, 4, 5, 6, 7, 8, and 9). Particularly, Temora turbinata, Calanus sinicus, Canthocalanus pauper, Undinula vulgaris and Paracalanus aculeatus were the five dominant species, comprising $81 \%$ of the total copepod numbers from NPP I and II during the monitoring period between November 2000 and December 2003. The present study shows for the first time the long term patterns of planktonic copepod assemblages (Tables 1 and 2) and spatial and temporal distribution of dominant species (Figures 3, 4, 5, 6, 7, 8, and 9) in the waters of NPP I and II. This information will be important for studying the copepod dynamics. Particularly, Most cruises were dominated by a few species (Table 2), with a large number of other species with very low density similar to Lo et al. [36]. This indicated that dominant copepod species may play a major role in the transfer of matters and energy in the adjacent waters of NPP I and NPP II. Furthermore, Calanus sinicus may transport from Bohai Sea, Yellow Sea and East China Sea during winter and spring into the waters of NPP I and II (Tables 1, 2, Figures 2, 3, 4, 5, 6, and 9) [26].

Several previous reports indicated that there was some side effect of the thermal discharge of power plants. The similar side effect also observed in the low copepod density very close to outlet waters of NPP I and II. However, this side effect usually occurred in a small scale of ocean e.g. within $500 \mathrm{~m}$ near the outlet water of 
NPP I and NPP II [18-23]. In large scale of ocean, the copepod species numbers, composition, and abundance in the waters adjacent to NPP I and NPP II (Tables 1 and 2 ) are within the ranges of many previous studies in the waters around Taiwan [1, 9-12, 16-17, 25, 28, 33-36, 40, 53]. It seems that the NE monsoon has much great influence on the copepod assemblages than the thermal discharge from NPP I and II.

\section{ACKNOWLEDGEMENTS}

We are grateful to Taiwan Power Company and National Museum of Marine Biology and Aquarium, Taiwan, ROC for their financial supports to J. S. Hwang. The authors acknowledge the long term helps from the captain and crews of Ocean Research Vessel II, NSC, Taiwan and sampling and technical helps from the laboratory members, W. S. Liao, C. M. Lin, Y. C. Chang, W. C. Lu, and C. L. Hsu of J.S. Hwang's. We also acknowledge the National Center for Ocean Research providing useful satellite data. We are grateful for the administrative helpers from Taiwan Power Company, C.-S. Tsai, C.-S. Guo, Y.-M. Lin, and G.K. Chen. We also thank the National Center of Scientific Research (CNRS) of France who supported the scientific stay of J.S. Hwang in France.

\section{REFERENCES}

1. Chang, W.-B. and Fang, L.-S., “Temporal and Spatial Variations of the Species Composition, Distribution, and Abundance of Copepods in Kaohsiung Harbor, Taiwan," Zool. Stud., Vol. 43, No. 2, pp. 454-463 (2004).

2. Chen, Q.-C., Zooplankton of China Seas (1), Science Press, Beijing, China, pp. 1- 87 (1992).

3. Chen, H.-Y. and Chen, Y.-L.L., "Quantity and quality of Summer Surface Net Zooplankton in the Kuroshio Current-Induced Upwelling Northeast of Taiwan," Terrest. Atmosph. Ocean. Sci., Vol. 3, pp. 321-334 (1992).

4. Chen, Q.-C. and Hwang, J.-S., "A New Species of Tortanus (Copepoda, Calanoida) from Taiwan," Crustaceana, Vol. 72, No. 3, pp. 265-271 (1999).

5. Chen, Q.-C., Hwang, J.-S., and Yin, J.-J., "A New Species of Tortanus (Copepoda, Calanoida) from Zhubi Reef Nansha (Spratly) Archipelagos," Crustaceana, Vol. 77, No. 2, pp. 129-135 (2004).

6. Cironi, R., Ioannilli, E., and Vitali, R., "Assessment of Effects of Coastal Power Plants on Marine Biological Resources in Italy," Coastal Ocean Space Utilization 3, Croce, N.D., Connell, S., and Abel, R. (Eds.), E and FN Spon, London, UK, pp. 313-329 (1995).

7. Fan, K.-L., "The Thermal Discharges from Nuclear Power Plants," Chem. Ecol., Vol. 6, pp. 213-224 (1992).

8. Guseva, V.P. and Chebotina, M.Y., "Radioecological
Studies on the plankton of the Cooling Water Reservoir of the Beloyarsk Nuclear Power Plant," Russian J. Ecol., Vol. 32, No. 4, pp. 249-254 (2001).

9. Hsiao, S.-H., Lee, C.-Y. Shih, C.-T., and Hwang, J.-S., "Calanoid Copepods of the Kuroshio Current East of Taiwan, with Notes on the Presence of Calanus Jashnovi Hulsemann, 1994," Zool. Stud., Vol. 43, No. 2, pp. 323331 (2004).

10. Hsieh, C.-H. and Chiu, T.-S., "Abundance Composition of the Copepods in Tanshui River Estuary and Adjacent Waters," Acta Zool. Taiwanica, Vol. 9, pp. 1-9 (1998).

11. Hsieh, C.-H. and Chiu, T.-S., "Summer Spatial Distribution of Copepods and Fish Larvae in Relation to Hydrography in the Northern Taiwan Strait," Zool. Stud., Vol. 41, pp. 85-98 (2002).

12. Hsieh C.-H., Chiu T.-S., and Shih, C.-T., "Copepod Diversity and Composition as Indicators of Intrusion of the Kuroshio Branch Current Into the Northern Taiwan Strait in Spring, 2000," Zool. Stud., Vol. 43, No. 2, pp. 393-403 (2004).

13. Hung, T.-C., Chiang, Y.-M., Tan, T.-H., Shao, K.-T., Huang, C.-C., Chen, J.-C., Chu, T.-C., and Fan, K.-L., "An Ecological Survey on the Waters Adjacent to the Northern Taiwan Nuclear Power Plant Sites Including Yenliao Coastal Area," SCOPE/Academia Sinica, Vol. 90, pp. 1-95 (1994).

14. Hung, T.-C., Fan, K.-L., Chiang, Y.-M., Tan, T.-H., Chang, K.-H., Shao, K.-T., Chu, T.-C., and Su, J.-C. "The Assessment of Ecological Impact on the Operation of the Nuclear Power Plants Along the Northern Coast of Taiwan (July 1974-June 1992)," SCOPE/Academia Sinica, Vol. 91, pp. 1-193 (1992).

15. Hung, T.-C., Huang, C.-C., and Shao, K.-T., "Ecological Survey of Coastal Water Adjacent to Nuclear Power Plants in Taiwan," Chem. Ecol., Vol. 15, pp. 129-142 (1998).

16. Hwang, J.-S., Chen, Q.-C., and Wong, C.-K., "Taxonomic Composition and Grazing Rate of Calanoid Copepods in Coastal Waters of Northern Taiwan," Crustaceana, Vol. 71, pp. 378-389 (1998).

17. Hwang, J.-S., Chen, Q.-C., and Wong, C.-K., "Taxonomic Composition, Density and Biomass of Free-Living Copepods in the Coastal Waters of Southwestern Taiwan," Crustaceana, Vol. 76, pp. 193-206 (2003).

18. Hwang, J.-S., Cheng, I.-J., Shao, K.-T., Hu, J.-H., Huh, C.-A., Fang, T.-H., and Lo, W.-T., "The Ecological Survey of Nuclear Power Plant I, II, and Pre-Operation IV, Northern Taiwan (Biological Parameters): Zooplankton," Annual Report of 2002, National Taiwan Ocean University, Keelung, Taiwan, pp. 4.1-4.57 (2003).

19. Hwang, J.-S., Shao, K.-T., Cheng, I.-J., and Lo, W.-T., "The Ecological Survey of Nuclear Power Plant I, II, and Pre-Operation IV, Northern Taiwan (Biological Parameters): Zooplankton," Annual Report of 1998, 
National Taiwan Ocean University, Keelung, Taiwan, pp. 4.1-4.15 (1999). (In Chinese with English Abstract).

20. Hwang, J.-S., Shao, K.-T., Cheng, I.-J., and Lo, W.-T., The Ecological Survey of Nuclear Power Plant I, II, and Pre-operation IV, Northern Taiwan (Biological Parameters): Zooplankton, Annual Report of 1999, National Taiwan Ocean University, Keelung, Taiwan, pp. 4.1-4.31 (2000). (In Chinese with English Abstract).

21. Hwang, J.-S., Shao, K.-T., Cheng, I.-J., and Lo, W.-T., The Ecological Survey of Nuclear Power Plant I, II, and Pre-operation IV, Northern Taiwan (Biological Parameters): Zooplankton, Annual Report of 2000, National Taiwan Ocean University, Keelung, Taiwan, pp. 4.1-4. 39 (2001). (In Chinese with English Abstract).

22. Hwang, J.-S., Shao, K.-T., Cheng, I.-J., Huh, C.-A., Hu, J.-H., Fang, T.-H., and Lo, W.-T., The Ecological Survey of Nuclear Power Plant I, II, and Pre-operation IV, Northern Taiwan (Biological Parameters): Zooplankton, Annual Report of 2001, National Taiwan Ocean University, Keelung, Taiwan, pp. 4.1-4.66 (2002).

23. Hwang, J.-S., Shao, K.-T., Cheng, I.-J., Hu, J.-H., Huh, C.-A., Fang, T.-H., and Lo, W.-T., The Ecological Survey of Nuclear Power Plant I, II, and Pre-operation IV, Northern Taiwan (Biological Parameters): Zooplankton, Annual Report of 2003, National Taiwan Ocean University, Keelung, Taiwan, pp. 4.1-4.73 (2004).

24. Hwang,J.-S. and Turner, J.T., "Behavior of Cyclopoid, Harpacticoid and Calanoid Copepods from Coastal Waters of Taiwan," Mar. Ecol., Vol. 16, No. 3, pp. 207216 (1995).

25. Hwang, J.-S., Wang, C.-H., and Chan, T.-Y. (eds.), Proceedings of the International Symposium on Marine Biology in Taiwan-Crustacean and Zooplankton Taxonomy, Ecology and Living Resources, Nat. Taiwan Museum Special Publ. Ser., No. 10, pp. 1-200 (2000).

26. Hwang, J.-S. and Wong, C.-K., "The China Coastal Current as a Driving Force for Transporting Calanus Sinicus (Copepoda: Calanoida) from Its Population Centers to Waters of Taiwan and Hong Kong During the NE Monsoon Period in Winter," J. Plankton Res., Vol. 27, No. 2, pp. 205-210 (2005).

27. Jan, S., Wang, J., Chern, C.-S., and Chao, S.-Y., "Seasonal Variation of the Circulation in the Taiwan Strait," J. Mar. Syst., Vol. 35, pp. 249-268 (2002).

28. Lan, Y.-C., Shih, C.-T., Lee M.-A., and Shieh, H.-Z., "Spring Distribution of Copepods in Relation to Water Masses in the Northern Taiwan Strait," Zool. Stud., Vol. 43, No. 2, pp. 332-343 (2004).

29. Lee, H.-J. and Chao, S.-Y., "A Climatological Description of Circulation in and around the East China Sea," Deep-Sea Res. II, Vol. 50, pp. 1065-1084 (2003).

30. Liang, W.-D., Tang, T.Y., Yang, Y.J., Ko, M.T., and Chuang, W.-S., "Upper-Ocean Currents around Taiwan," Deep-Sea Res. II, Vol. 50, pp. 1085-1106
(2003).

31. Lin, Y.-H., "The Relation Between Malformed Fishes and the Temperature of Thermal Discharges at the $2^{\text {nd }}$ Nuclear Power Plant on the Northern Coast of Taiwan," M.S. Thesis, Institute of Marine Biology, National Taiwan Ocean University, Keelung, Taiwan (1995).

32. Liu, K.-K., Peng, T.-H., Shaw, P.-T., and Shiah, F.-K., "Circulation and Biogeochemical Processes in the East China Sea and the Vicinity of Taiwan: An Overview and a Brief Synthesis," Deep-Sea Res. II, Vol. 50, pp. 10551064 (2003).

33. Lo, W.-T., Chung, C.-L., and Shih, C.-T., "Seasonal Distribution of Copepods in Tapong Bay, Southwestern Taiwan," Zool. Stud. Vol. 43, No. 2, pp. 464-474 (2004).

34. Lo W.-T., Hwang J.-S., and Chen Q.-C., "Identity and Abundance of Surface Dwelling, Coastal Copepods of Southwestern Taiwan," Crustaceana, Vol. 74, No. 10, pp. 1139-1157 (2001).

35. Lo, W.-T., Hwang, J.-S., and Chen, Q.-C., "Spatial Distribution of Copepods in Surface Waters of the Southeastern Taiwan Strait," Zool. Stud., Vol. 43, No. 2, pp. 218-228 (2004).

36. Lo, W.-T., Shih, C.-T., and Hwang, J.-S., "Diel Vertical Migration of the Planktonic Copepods at an Upwelling Station North of Taiwan, Western North Pacific," J. Plankton Res., Vol. 26, No. 1, pp. 89-97 (2004).

37. Pu, X.-M., Sun, S., Yang, B., Ji, P., Zhang, Y.-S., and Zhang, F., "The Combined Effects of Temperature and Food Supply on Calanus Sinicus in the Southern Yellow Sea in Summer," J. Plankton Res., Vol. 26, No. 9, pp. 1049-1057 (2004).

38. Pu, X.-M., Sun, S., Yang, B., Zhang, G.-T., and Zhang, F., "Life History Strategies of Calanus sinicus in the Southern Yellow Sea in Summer,' J. Plankton Res., Vol. 26, No. 9, pp. 1059-1068 (2004).

39. Qiongbei, J., Lianxi, S., and Ran, Z., "Effects of Thermal Discharge from a Power Plant on Zooplanktons," Acta Scientiae Circumstantiate, Vol. 9, No. 2, pp. 208-217 (In Chinese with English Abstract), (1989).

40. Shih, C.-T. and Chiu, T.-S., "Copepod Diversity in the Water Masses of the Southern East China Sea North of Taiwan," J. Mar. Syst., Vol. 15, pp. 533-542 (1998).

41. Shih, C.-T. and Young, S.-S., "A Checklist of FreeLiving Copepods, Including Those Associated with Invertebrates Reported from the Adjacent Seas of Taiwan," Acta Zool. Taiwanica. Vol. 6, pp. 65-81 (1995).

42. Su, J.-C., Hung, T.-C., Chiang, Y.-M., Tan, T.-H., Chang, K.-H., Lee, C.-T., and Chang, H.-T., The Preliminary Report of the Assessment of Ecological Impact of the Operation of the First Nuclear Power Plant Along the Northern Coast of Taiwan (July 1974-June 1981), Special Publication, Atomic Energy Council, Taipei, Taiwan, pp. 1-89 (1981).

43. Su, J.-C., Hung, T.-C., Chiang, Y.-M., Tan, T.-H., Chang, 
K.-H., Lee, C.-T., and Chang, H.-T., The Final Report for the Assessment of Ecological Impact on the Operation of the Nuclear Power Plants Along the Northern Coast of Taiwan (July 1974-June 1984), SCOPE/ Academia Sinica, Vol. 29, pp. 1-151 (1984).

44. Su, J.-C., Hung, T.-C., Chiang, Y.-M., Tan, T.-H., Lo, C.-T., Chang, K.-H., Lee, C.-T., and Chang, H.-T., An Ecological Survey on the Waters Adjacent to the Northern Taiwan Nuclear Power Plant Sites, Background Studies (July 1974-June 1979), Special Publication, Atomic Energy Council, Taipei, Taiwan (1979).

45. Suresh, K., Durairaj, G., and Nair, K.V.K., "Harpacticoid Copepod Distribution on a Sandy Shore in the Vicinity of a Power Plant Discharge, at Kalpakkam, Along the East Coast of India," Indian J. Mar. Sci., Vol. 25, pp. 307-311 (1996).

46. Tomasino, M., Ambrogi, R., and Ioannilli, E., "Scientific Approach for Evaluating the Sites of Coastal Thermoelectric Power Stations," Coastal Ocean Space Utilization 3, Croce, N.D., Connell, S., and Abel, R. (Eds.), E \& FN Spon, London, UK, pp. 197-211 (1995).

47. Tseng, R.-S. and Shen, Y.-T., "Lagrangian Observations of Surface Flow Patterns in the Vicinity of Taiwan," Deep-Sea Res. II, Vol. 50, pp. 1107-1116 (2003).

48. Uye, S., "Temperature-dependent Development and Growth of Calanus Sinicus (Copepoda: Calanoida) in the Laboratory," Hydrobiologia, Vol. 167/168, pp. 285293 (1988).

49. Uye, S., "Why does Calanus Sinicus Prosper in the Shelf Ecosystem of the Northwest Pacific Ocean?" ICES J. Mar. Sci., Vol. 57, pp. 1850-1855 (2000).
50. Vandish, O.I., "Zooplankton as an Indicator of Lake Ecosystem Conditions: Case Study of Subarctic Lake Imandra," Water Resour., Vol. 27, No. 3, pp. 364-370 (2000).

51. Vranovsky, M., "Impact of the Gabcikovo Hydropower Plant Operation on Planktonic Copepods Assemblages in the River Danube and its Floodplain Downstream of Bratislava," Hydrobiologia, Vol. 347, pp. 41-49 (1997).

52. Wang, R., Zuo, T., and Wang, K., "The Yellow Sea Cold Bottom Water-an Oversummering Site for Calanus Sinicus (Copepoda, Crustacea)," J. Plankton Res., Vol. 25, pp. 169-183 (2003).

53. Wong, C.-K., Hwang, J.-S., and Chen, Q.-C., “Taxonomic Composition and Grazing Impact of Calanoid Copepods in Coastal Waters Near Nuclear Power Plants in Northern Taiwan," Zool. Stud., Vol. 37, No. 4, pp. 330-339 (1998).

54. Wong, G.T.F., Chao, S.-Y., Li, Y.-H., and Shiah, F.-K., "The Kuroshio Edge Exchange Processes (KEEP) Studyan Introduction to Hypotheses and Highlights," Cont. Shelf Res., Vol. 20, pp. 335-347 (2000).

55. Wu, C.-H., Hwang, J.-S., and Yang, J.-S., "Diets of Three Copepods (Poecilostomatoida) in the Southern Taiwan Strait," Zool. Stud., Vol. 43, No. 2, pp. 388-392 (2004).

56. Yang, Y.-F., Wang, Z.-D., Pan, M.-X., and Jiao, N.-Z., "Zooplankton Community Structure of the Sea Surface Microlayer Near Nuclear Power Plants and Marine Fish Culture Zones in Daya Bay," Chinese J. Oceanol. Limnol., Vol. 20, No. 2, pp. 129-134 (2002). 